\title{
Slaying Monster with Catheter: A Case Report
}

\author{
Mohd Saad Jalaluddin @ Khin Maung Zan¹, Shaiful Azmi Yahaya¹, Al-Fazir Omar² \\ ${ }^{1}$ National Heart Institute, Malaysia \\ ${ }^{2}$ Cardiac Vascular Sentral, Kuala Lumpur
}

Main Author: Mohd Saad Jalaluddin Khin Maung Zan

Email: mohdsaad008@gmail.com

\section{SUMMARY}

This is a case of successful treatment of secondary hypertension with catheter intervention in a 62 years old gentleman who has severe aortoiliac occlusive disease, severe coronary artery disease and other comorbidities. Early recognition, diagnosis and treatment of secondary causes of hypertension lead to good clinical outcomes, possible reversal of end-organ damage and better blood pressure control. Blood pressure control is essential prior to coronary intervention in patients with resistant hypertension and concomitant coronary artery disease to prevent haemorrhagic stroke and other unwanted complications. This gentleman has triple vessels and left main stem disease which surgeons refused for coronary artery by pass graft (CABG) in view patient has severe aorto-iliac occlusive disease with resistant hypertension. We have done successful revascularization to aorto-iliac followed by coronary intervention.

\section{KEYWORDS}

secondary/resistant hypertension, coronary artery disease, aortoiliac occlusive disease, catheter (endovascular) intervention.

\section{INTRODUCTION}

Severe aortoiliac stenosis or occlusion is most often a chronic disease resulting from progressive atherosclerotic and thrombotic accumulation. ${ }^{1,5}$ All patients presenting with symptoms related to aortoiliac disease should undergo a thorough history and physical examination. ${ }^{1,4}$ However, presence of collaterals allows many patients to present with significantly less severe symptoms than what one might expect from such an advanced disease process. ${ }^{3}$ As many as $90 \%$ of these patients have a smoking history. These group of patients also have high rate of hypertension (65-87\%), coronary artery disease (30-60\%), chronic obstructive pulmonary disease (13-20\%) and chronic renal insufficiency (4-13\%).

Patients with aortoiliac occlusive disease have a 2.5-3.5 folds risk of mortality and cardiovascular events compared with those with distal peripheral arterial disease. ${ }^{2}$ Interventional therapies can benefit patients with secondary hypertension significantly in respect of mortality and quality of life. ${ }^{7,9}$ Endovascular therapies should be considered as first-line therapy for all patients with aortoiliac occlusive disease over open surgical approaches with increasing experience, technical skill and improved endovascular equipment. ${ }^{6,10}$

\section{CASE REPORT}

We describe Mr. NMN, a 62-year-old gentleman with multiple comorbidities. He has resistant hypertension, dyslipidaemia, previous history of ischemic stroke, chronic hepatitis B infection and stage 3 chronic kidney disease.

He presented with progressive shortness of breath and treated for acute pulmonary oedema secondary to hypertensive emergency. He was also treated for NSTEMI in the ward as he had chest pain with dynamic ECG changes and raised troponin. Attempted coronary angiogram via right and left femoral artery approach was unsuccessful as we noted that there was occluded right and left common iliac arteries. Coronary angiogram was performed via right radial approach and noted to have triple vessel with distal left main stem (LMS) disease.

Patient was referred to cardiothoracic team for Coronary artery Bypass Graft (CABG). Surgeon decided not for CABG in view of resistant hypertension with aortoiliac occlusive disease. We proceed with CT angiogram and it showed complete occlusion of abdominal aorta after Superior Mesenteric Artery (SMA). There is reconstitution of right and left external iliac arteries via collaterals mainly from SMA, inferior epigastric and deep circumflex iliac arteries. The right and left distal Common Femoral Artery (CFA) and Superficial Femoral Arteries (SFA) are normal and demonstrates good blood flow. The right and left main renal arteries are reconstituted from collaterals. There is poor perfusion of the posterior inferior part of left kidney. He was then referred to vascular surgeon for bilateral aortoiliac bypass but was turned down.

His systolic blood pressure was persistently $>200 \mathrm{mmHg}$ despite on 7 anti-hypertensive medications. He was having intermittent chest pain in the ward with ECG showing extensive ST depression and T wave inversion. Echocardiogram showed LVEF 53\% with concentric left ventricular hypertrophy and normal valves.

We decided to control the blood pressure first via catheter intervention of aortoiliac disease prior to coronary intervention. His blood pressure was recorded as $214 / 98 \mathrm{mmHg}$ prior to the procedure. We proceeded with Percutaneous Transluminal Angioplasty (PTA) of aortoiliac through left brachial artery, right femoral artery and left femoral artery punctures with 6FR sheaths. 
Figure 1a: severe stenosis at mid left circumflex artery (LCx). Figure 1b: severe distal LMS disease with severe disease proximal to mid left anterior descending artery (LAD).

Figure 1c: moderate disease mid right coronary artery (RCA) and severe disease at distal RCA.

Figure 1d: Occluded right common iliac artery

Figure 1e: Occluded left common iliac artery

Figure 1f: CT angiogram surprised us as it showed complete occlusion of abdominal aorta after Superior Mesenteric Artery (SMA).

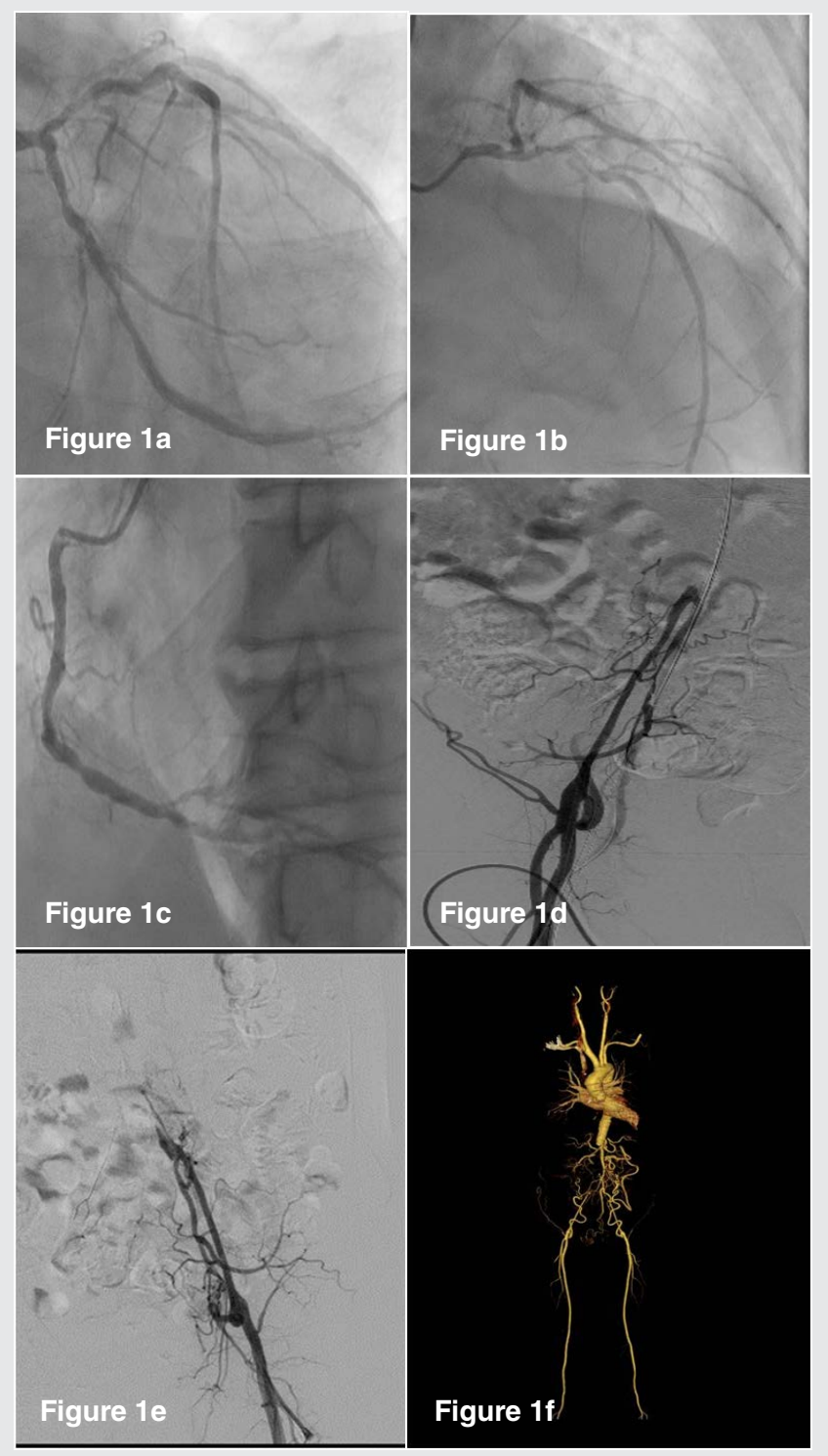

Attempted to cross antegrade approach with Terumo stiff wire and JR 3.5 6F support but wire went into false lumen. We switched to retrograde approach via right femoral access and managed to cross with command 0.014 wire using Subintimal Arterial Flossing with antegrade-retrograde intervention (SAFARI) technique. Ballooning was done with Admiral Xtreme $4.0 \times 60$ $\mathrm{mm}, 5.0 \times 20 \mathrm{~mm}$ and then $7.0 \times 60 \mathrm{~mm}$ balloons. Subsequently wire was externalized to right femoral artery. We stented infra SMA into right external iliac and right common femoral artery with EPIC 14/60/7.0 mm, ABSOLUTE PRO LL $7.0 \times 150 \mathrm{~mm}$ and
VASCUFLEX $7.0 \times 80 \mathrm{~mm}$. Post dilated with Comral Xtreme 7.0 $\times 60 \mathrm{~mm}$. We achieved good flow into the right femoral artery. However, we failed to cross left common iliac artery.

Figure 2a: Subintimal Arterial Flossing with antegraderetrograde intervention (SAFARI) technique

Figure $\mathbf{2 b}, \mathbf{2 c}$ and $\mathbf{2 d}$ : angiogram pictures showing post angioplasty to aortoiliac.

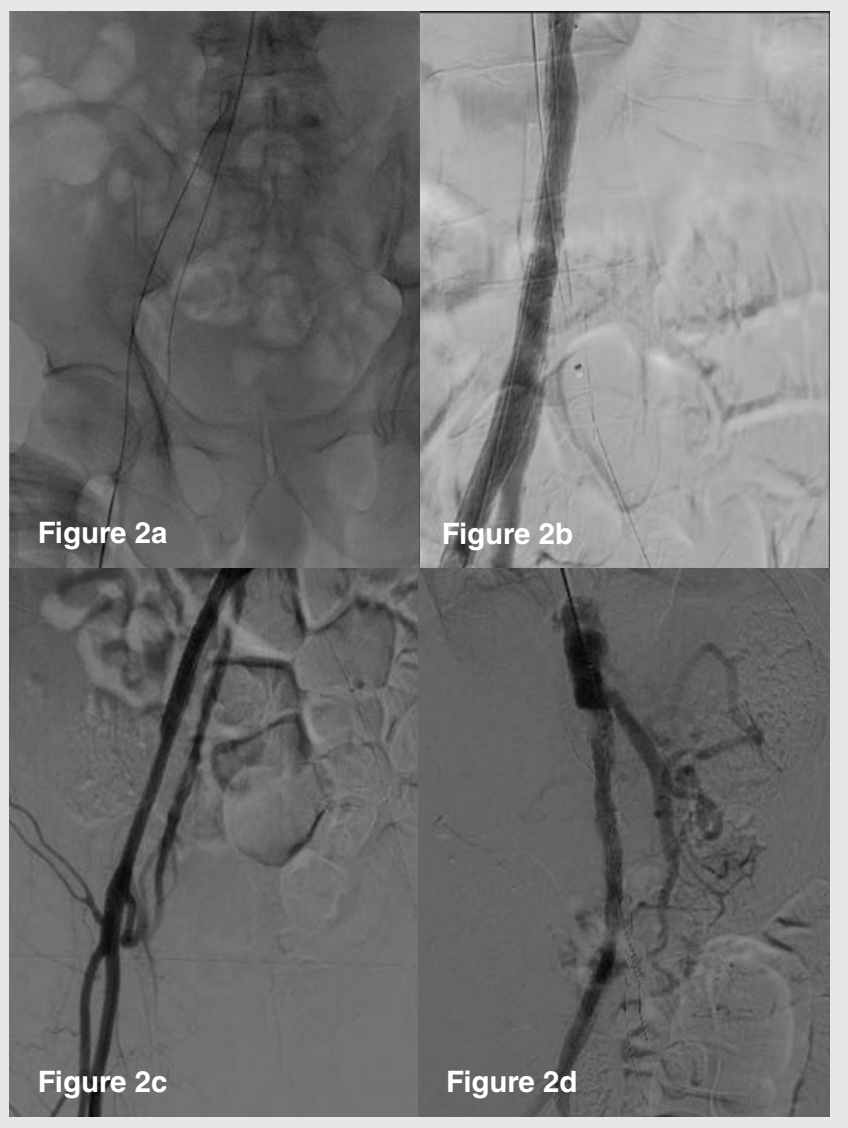

Patient's blood pressure has significantly come down to 150/90 $\mathrm{mmHg}$ soon after the procedure. He developed contrast induced nephropathy but improved without dialysis. We managed to reduce his number of antihypertensives to 4 from 7 .

He developed NSTEMI in ward again with ECG showing anterior ischemia and raised cardiac markers. Repeat echocardiography showed further drop in EF 38\% from 53\%. A family conference was done and discussed about high risk angioplasty to $L M / L A D /$ LCx and medical therapy to RCA.

We proceeded with angioplasty to LCx via right radial artery approach with 6 FR sheath, EBU 3.5/6FR guiding catheter and run through floppy wire. Pre-dilated LCx lesion with Emerge 2.5 $\times 15 \mathrm{~mm}$ and stented with Biomatrix Alpha $3.0 \times 19 \mathrm{~mm}$. post dilatation was done with NC Quantum Apex $3.0 \times 12 \mathrm{~mm}$ at 24 atm.

Angioplasty to LM-LAD was done with provisional single stent technique. The lesion was pre-dilated with NC Quantum Apex 3.0 $x 12 \mathrm{~mm}$ at $16 \mathrm{~atm}$ and NSE Alpha $3.5 \times 13 \mathrm{~mm}$ at $14 \mathrm{~atm}$. We 
were unable to deliver a long stent due to calcified and tortuous proximal LAD. Hence, we decided to use 2 stents strategy. The lesion was stented with Resolute Onyx $3.5 \times 18 \mathrm{~mm}$ and $3.5 \times 26$ $\mathrm{mm}$. it was post dilated with NC Euphora $4.0 \times 8 \mathrm{~mm}$ at $20 \mathrm{~atm}$. IVUS was done post procedure and it showed that stent was well apposed. It was a successful result with TIMI 3 flow. Patient was discharged well 3 days after the procedure.

Figure 3a: post angioplasty to LCX

Figure 3b: post angioplasty to LM-LAD

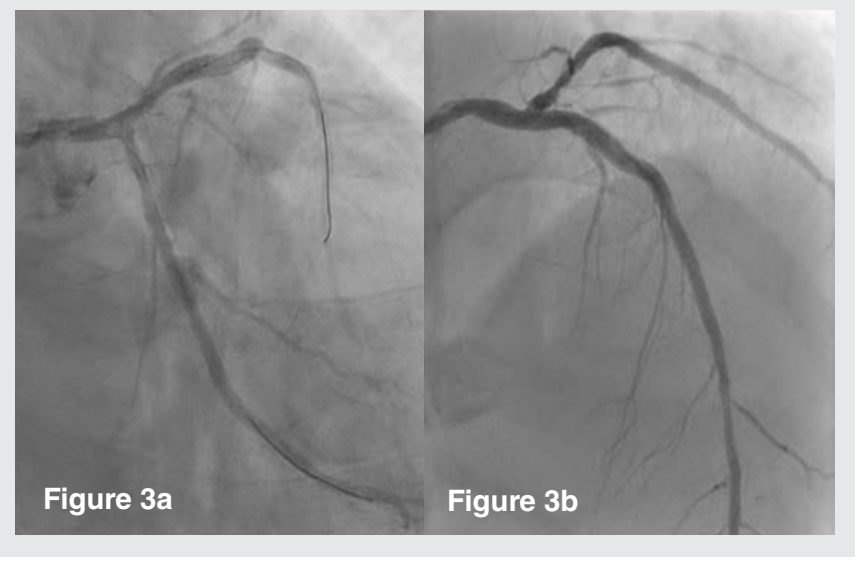

\section{DISCuSSION}

This patient was diagnosed late for probable cause of resistant hypertension despite on multiple antihypertensive medications. It is important to recognize secondary causes of hypertension as they may be curable. If left undiagnosed, it can lead to resistant hypertension, cardiovascular and renal complications, multiple specialist referrals and unnecessary burden on the healthcare system. Even though this patient has developed collaterals with no evidence of claudication, it was still insufficient for good renal perfusion and causing resistant hypertension.

Treatment of severe aortoiliac disease has dramatically evolved from a dependence on open aortobifemoral grafting to hybrid and endovascular only approaches. $6,8,9,10$ Open surgery has been the gold standard treatment of severe aortoiliac disease with excellent patency rates, but with increased length of stay and major complications. In contrast, endovascular interventions can successfully treat almost any lesion with decreased risk, compared to open surgery. ${ }^{10}$ Although primary patency rates remain inferior, secondary endovascular interventions are often minor procedures resulting in comparable long-term outcomes. ${ }^{7}$.

${ }^{10}$ The risks of renal insufficiency, embolization and access complications are not insignificant; however, most can be prevented or managed without significant clinical consequence. ${ }^{7,8}$

In conclusion, we have successfully managed to treat the secondary cause of hypertension for this gentleman via catheter intervention. We proceeded with peripheral intervention first in order to bring down the blood pressure followed by coronary intervention. Blood pressure control was essential to reduce the risk of haemorrhagic stroke during angioplasty. As we failed to cross left common iliac artery, either reattempt endovascular intervention or femoral bypass will benefit if patient agreeable.

\section{REFERENCES}

1. Poulter NR, Prabhakaran D, Caulfield M.Hypertension. Lancet 2015;386:801-12.

2. Pedrosa RP, Drager LF, Gonzaga CC, et al. Obstructive sleep apnea: the most common secondary cause of hypertension associated with resistant hypertension. Hypertension. 2011;58:811-7.

3. Cooper CJ, Murphy TP, Cutlip DE, et al. C ORAL Investigators Stenting and medical therapy for atherosclerotic renal-artery stenosis. N Engl J Med. 2014:370:13-22.

4. Davis BA, Crook JE, Vestal RE, Oates JA. Prevalence of renovascular hypertension in patients with grade III or IV hypertensive retinopathy. $N$ Engl J Med 1979; 301:1273.

5. Hansen KJ, Edwards MS, Craven TE, et al. Prevalence of renovascular disease in the elderly: a population-based study. J Vasc Surg. 2002; 36:443.

6. Rooke TW, Hirsch AT, Misra S, et al. 2011 ACCF/AHA Focused Update of the Guideline for the Management of Patients with Peripheral Artery Disease (updating the 2005 guideline): a report of the American College of Cardiology Foundation/American Heart Association Task Force on Practice Guidelines. J Am Coll Cardiol. 2011; 58:2020.

7. Ritchie J, Green D, Chrysochou C, et al. High-risk clinical presentations in atherosclerotic renovascular disease: prognosis and response to renal artery revascularization. Am J Kidney Dis. 2014;63:186.

8. Norgren L, Hiatt WR, Dormandy JA, et al. Inter-Society Consensus for the Management of Peripheral Arterial Disease (TASC II) J Vasc Surg. 2007;45(Suppl S):S5-67. [Updated international working group consensus for the management of peripheral arterial disease.]

9. Ligush J, Criado E, Burnham SJ, et al. Management and outcome of chronic atherosclerotic infrarenal aortic occlusion. J Vasc Surg. 1996;24(3):394-404. discussion 404-405.

10. Dimick JB, Cowan JA, Henke PK, et al. Hospital volume-related differences in aorto-bifemoral bypass operative mortality in the United States. $J$ Vasc Surg. 2003;37(5):970-5. 